\title{
Redescripción del zapotillo rosado del Perú, Phragmotheca sidereotricha Fern. Alonso (Malvaceae), y notas sobre su hábitat y distribución
}

\author{
José Luis Fernández-Alonso,*, Robin Fernández-Hilario², Carlos Reynel-Rodríguez² \\ ${ }^{1}$ Real Jardín Botánico, Consejo Superior de Investigaciones Científicas, Madrid, España \\ ${ }^{2}$ Herbario Forestal, Universidad Nacional Agraria La Molina, Facultad de Ciencias Forestales, Lima, Perú
}

\begin{abstract}
Resumen
Se presenta la descripción completa del árbol endémico del Perú conocido como zapotillo rosado (Phragmotheca sidereotrica Fern. Alonso, Malvaceae), elaborada con base en las nuevas recolecciones estudiadas, cuyo material se encuentra en los siguientes herbarios: Museo Herbario Amazonense (AMAZ), Herbario Nacional Colombiano (COL), Herbario Selva Central-Oxapampa (HOXA), Herbario Sur Peruano (HSP), Herbario del Real Jardín Botánico (MA), Herbario del Jardín Botánico de Missouri (MO), Herbario de la Universidad Nacional Agraria La Molina (MOL) y el Herbario de la Universidad Nacional Mayor de San Marcos (USM). Se documenta su hábitat, ecología, distribución y categorización según la Unión Internacional para la Conservación de la Naturaleza (UICN), y se presenta el mapa de su distribución con las nuevas localidades registradas, todas ellas en el Departamento de Pasco, Provincia de Oxapampa, Distritos de Palcazú y Puerto Bermúdez. Se incluyen algunas fotografías de la flor y del fruto de este árbol y también una clave para diferenciar los cuatro géneros de frutos carnosos de la tribu Matisieae presentes en Perú. (C) 2017. Acad. Colomb. Cienc. Ex. Fis. Nat.
\end{abstract}

Palabras clave: Bombacoideae; Malvaceae; Matisieae; Perú; Phragmotheca; Sterculioideae.

Redescription of the pink zapotillo of Perú, Phragmotheca sidereotricha Fern. Alonso (Malvaceae), and notes on its habitat and distribution

\begin{abstract}
We present the complete description of the endemic tree of Perú known as zapotillo rosado, (Phragmotheca sidereotrica Fern. Alonso, Malvaceae), based on the latest collections studied, whose material is in the following herbaria: AMAZ, COL, HOXA, HSP, MA, MO, MOL and USM. We documented its habitat, ecology, distribution and categorization according to IUCN, and we present a distribution map with the new registered localities, all in the Department of Pasco, Province of Oxapampa, Districts of Palcazú and Puerto Bermúdez, Perú. We include photographs of its flowers and fruits, as well as a key to differentiate the four genera of fleshy fruits of the tribe Matisieae present in Perú. (C) 2017. Acad. Colomb. Cienc. Ex. Fis. Nat.
\end{abstract}

Key words: Bombacoideae; Malvaceae; Matisieae; Perú; Phragmotheca; Sterculioideae.

\section{Introducción}

El género Phragmotheca Cuatrec. incluye 11 especies distribuidas desde el este de Panamá, pasando por Colombia y Ecuador, hasta el centro de Perú (Alverson, 1991; FernándezAlonso, 1996; Fernández-Alonso, 1999). Junto con Matisia Bonpl., Quararibea Aubl. y otros ocho a nueve géneros menores, se ha considerado tradicionalmente como parte de la tribu Matisieae K. Schum. de la familia Bombacaceae, más recientemente tratada como subfamilia Bombacoideae de la familia Malvaceae s.l. (Fernández-Alonso, 1996; Bayer, et al., 1999; Fernández-Alonso, 2011, Angiosperm Phylogeny Group IV - APG IV, 2016).

En los primeros estudios moleculares de Malvaceae s.l., la tribu Matisieae no mostró una clara afinidad hacia las subfamilias Bombacoideae Burnet o Malvoideae Burnet
(Bayer, et al., 1999; Alverson, et al., 1999). Sin embargo, aunque en trabajos posteriores se ha sustentado la posición de la tribu Matisieae fuera del núcleo Bombacoideae, sigue sin decantarse su ubicación a nivel de subfamilia, bien en las Malvoideae o en las Sterculiodeae Beishmied con una circunscripción ampliada (Baum, et al., 2004; Nyffeler, et al., 2005, Duarte, et al., 2011; Carvalho-Sobrinho, et al., 2016; APG IV, 2016).

Durante trabajos de campo realizados en el 2014 en el Bosque de Protección San Matías - San Carlos, en la selva central de Perú, se registraron individuos de Phragmotheca

\footnotetext{
*Correspondencia:

José Luis Fernández-Alonso, jlfernandeza@rjb.csic.es

Recibido: 04 de julio de 2017

Aceptado: 25 de agosto de 2017
} 
con corolas de color rojo, carácter que solo había sido reportado para dos especies de Colombia. Después de revisar y analizar otros caracteres, se pudo confirmar que esta entidad correspondía a una especie muy poco conocida y originalmente descrita con flores amarillas, con lo cual se amplió su distribución. En el presente trabajo se hace una nueva descripción detallada de $P$. sidereotricha, se registran otras seis localidades nuevas para esta especie, sustentadas con registros de herbario, además de información crítica sobre la ecología de la tribu Matisieae.

\section{Tratamiento taxonómico}

Phragmotheca se caracteriza por su hábito arbóreo, con hojas simples, ovadas a cordadas; flores opositifolias, a veces más o menos zigomórficas, cáliz tubular a campanulado, ápice 3-5 lobado, persistente, a veces acrescente, cupular en el fruto, pétalos más o menos espatulados, blancos o amarillentos, menos frecuentemente rojos, androceo con tubo estaminal apicalmente 5-lobado, lóbulos alargados, cada uno con 3-6 anteras sésiles septadas, ovario 5-locular, lóculos 2ovulados, estigma capitado a lobado; frutos drupáceos, con exocarpo coriáceo, mesocarpo carnoso-fibroso, y con cinco pirenos con endocarpo leñoso y grueso, frecuentemente costillado; generalmente con 5 semillas o menos, y cotiledones aplanados o doblados (Fernández-Alonso, 1996; Bayer \& Kubitzki, 2003.

\section{Clave para diferenciar los géneros de la tribu Matisieae} de frutos drupáceos.

1. Cada lóculo del ovario (5) con numerosos óvulos. Fruto con cinco series de semillas globosas y pubescentes .... Patinoa Cuatrec.

1'. Cada lóculo del ovario (generalmente 5) con solo dos óvulos. Fruto con 2 a 5 pirenos córneos o leñosos, no pubescentes .... 2

2. Lóbulos del tubo estaminal de hasta $4 \mathrm{~mm}$ de longitud; ovario 2-4 locular y frutos con 1 a 4 pirenos desarrollados .. Quararibea Aubl.

2'. Lóbulos del tubo estaminal de más de 4 mm de longitud; ovario 5 locular y frutos con cinco pirenos desarrollados ..... 3

3. Hojas con indumento lepidoto, estrellado o fasciculado, anteras con tecas septadas, pirenos gruesos con costillas marcadas

Phragmotheca Cuatrec.

3'. Hojas con indumento estrellado o fasciculado (nunca lepidoto), anteras con tecas no septadas, pirenos generalmente delgados y siempre sin costillas marcadas...... Matisia Bonpl.

Phragmotheca sidereotricha Fern. Alonso, Caldasia 18(3): 279, fig 12. 1996. Figura 1.

Tipo. PERÚ. Dpto. Pasco, Prov. Oxapampa, Distrito Iscozacín [Distrito Palcazú, Centro Poblado de Iscozacín], 04 de junio de 1986, fl., W. Pariona, \& J. Quijano 55 (holotipo MO [MO-176518]; isotipo F [F0042736F]).

Descripción ampliada. Árbol de hasta $26 \mathrm{~cm}$ de diámetro y $20 \mathrm{~m}$ de alto, con el fuste ligeramente cilíndrico y la base recta; corteza externa marrón, lenticelada; corteza interna reticulada, de color amarillento a naranja. Ramitas terminales de color gris, con sección circular, de 4-5,5 mm de diámetro, rugosas cuando secas; las yemas ferrugíneas, con fino indumento estrellado-fasciculado. Hojas simples, alternas y dispuestas en espiral, con pecíolos de (7)10 25(35) x 1-2 mm, doblemente pulvinulados, el pulvínulo distal más manifiesto, en seco ocráceo, con indumento fino estrellado-fasciculado; láminas cartáceas de (6,6-) 8-18,2 $(-23,3) \mathrm{cm}$ de longitud por $(2,5-) 4-8 \mathrm{~cm}$ de ancho, elípticas a lanceoladas, rara vez oblongas u obovadas-elípticas, el ápice acuminado a obtuso, la base obtusa a redonda, el margen entero, con tres nervios basales y dos submarginales poco notorios, la nerviación ligeramente broquidódroma, los nervios secundarios de 4-7 pares, la nerviación terciaria reticulada y marcada en el envés, haz y envés con indumento de pelos estrellados dispersos, más abundantes sobre los nervios, rara vez glabrescentes. Flores solitarias, generalmente opuestas a las hojas en ramas laterales cortas, con 2-3 bractéolas lineares, desiguales, de $2-4,5 \mathrm{~mm}$ de longitud, ubicadas en el tercio distal del pedicelo, prontamente caducas; pedicelo floral de (8)10-20 mm de longitud por cerca de 1,5 mm de diámetro, ferrugíneo; cáliz en botón fusiforme, apiculado, estrechamente tubular en la antesis, y desigualmente rasgado en la zona apical, de (16)18-20 $\mathrm{mm}$ de longitud por 5-6 $\mathrm{mm}$ de ancho, la cara externa ferrugínea, el interior adpreso-seríceo, los lóbulos obtusos de cerca de 4-5 mm de longitud; corola roja (referida como amarilla en una de las etiquetas de colección de herbario), los pétalos estrechamente espatulados, de (25)30-35 mm de longitud y (6)8-10,5 $\mathrm{mm}$ en la parte distal más ancha, con indumento generalmente estrellado (o fasciculado) disperso en las caras y con los márgenes ciliados; androceo con columna estaminal de (20)24-26 mm de longitud total, el tubo de $14-16 \mathrm{~mm}$ de longitud y cerca de $1 \mathrm{~mm}$ de diámetro, dispersamente ciliado en la parte media y basal, largamente ciliado en la parte distal (pelos multicelulares moniliformes), 5 ramas estaminales de cerca de $10 \mathrm{~mm}$ de longitud por 1-1,5 $\mathrm{mm}$ de ancho, planas en la cara interna, y largamente ciliadas (pelos multicelulares moniliformes), con 4-6 tecas por rama, 4 alargadas en la parte media y distal, de (2)3-3,5 mm de longitud con (8)9-13 lóculos por teca, y $0-2$ tecas pequeñas en la parte proximal, de $1-1,5$ $\mathrm{mm}$ de longitud con (2-) 5-8 lóculos por teca, los lóculos de 0,5-0,75 mm de longitud, dispuestos en dos series; gineceo con el estilo filiforme, 5-sulcado, de 21-22 mm de longitud total y cerca de $0,25 \mathrm{~mm}$ de diámetro en la parte media, los tres primeros cuartos glabros, el cuarto distal ciliado, curvo y de $1 \mathrm{~mm}$ de diámetro, el estigma capitado, de 1,5-2,0 $\mathrm{mm}$ de longitud, el ovario con 5 carpelos. Pedicelo fructífero cilíndrico, de 4,5-10 $\mathrm{mm}$ de longitud por $5 \mathrm{~mm}$ de ancho; cáliz fructífero pateliforme, de 1,9-2,5 $\mathrm{cm}$ de diámetro, con los márgenes rasgados irregularmente en el margen distal; frutos drupáceos, amarillos en la madurez, ovadoapiculados, de 4,6-5,9 $\mathrm{cm}$ de longitud por $2,3-3,5 \mathrm{~cm}$ de diámetro, el apículo de 1,4-1,7 $\mathrm{cm}$ de longitud, el exocarpo liso, marrón verdoso o amarillento, con fino indumento 


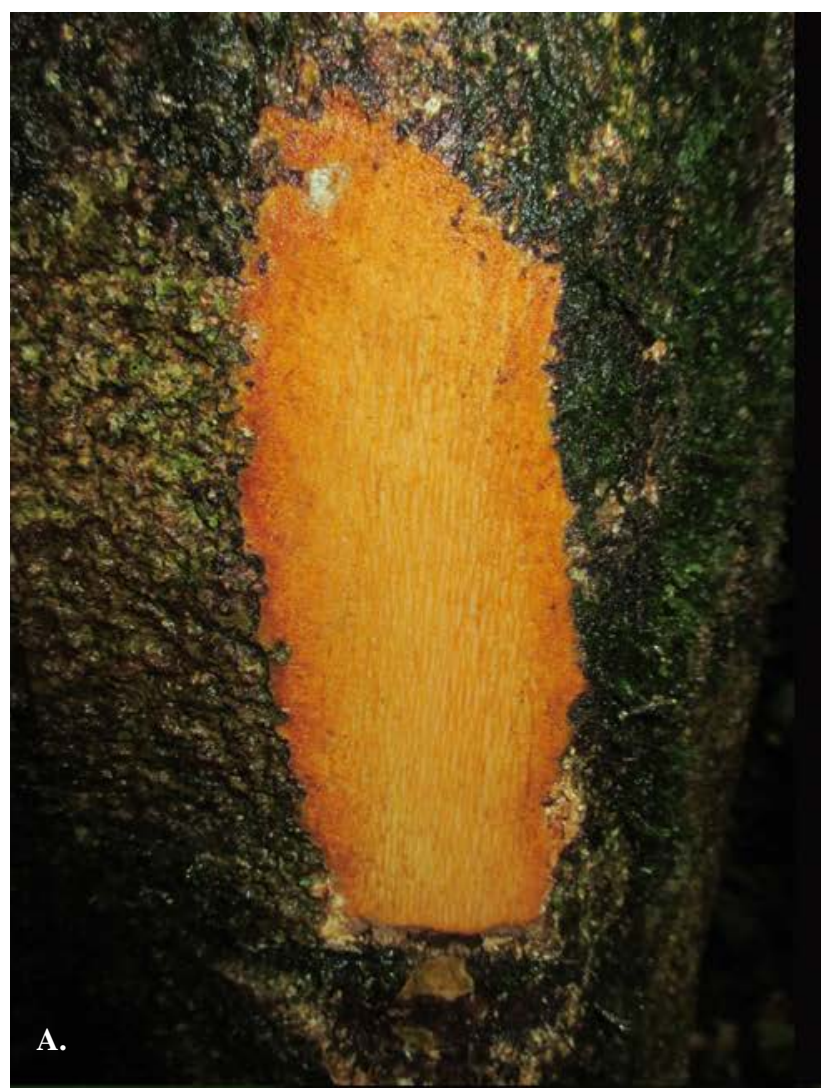

B.

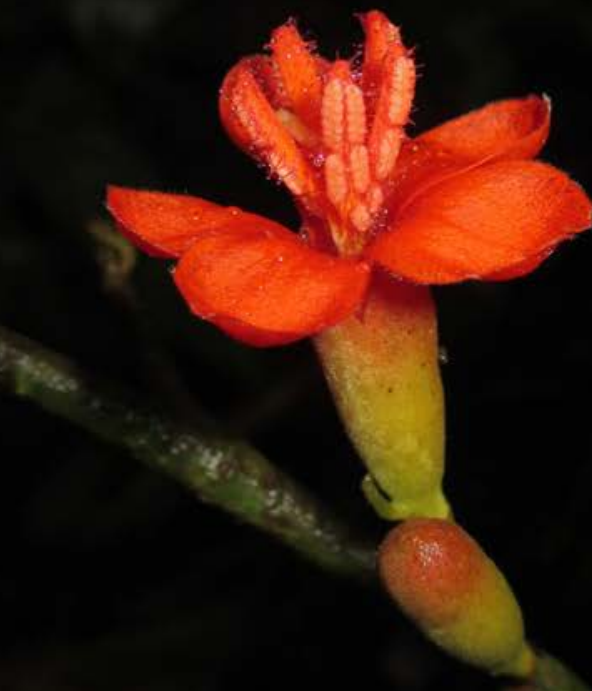

C.

Figura 1. Phragmotheca sidereotricha. A. Detalle de la corteza. B. Rama corta con botones florales con las bractéolas en la zona media y distal de los pedicelos. C. Flora abierta con los pétalos patentes y el androceo en posición central. D. Rama con fruto maduro en su posición natural con el apículo dirigido hacia abajo y con cáliz fructífero persistente, pateliforme en la parte inferior. (Fotografías de Robin Fernández). 


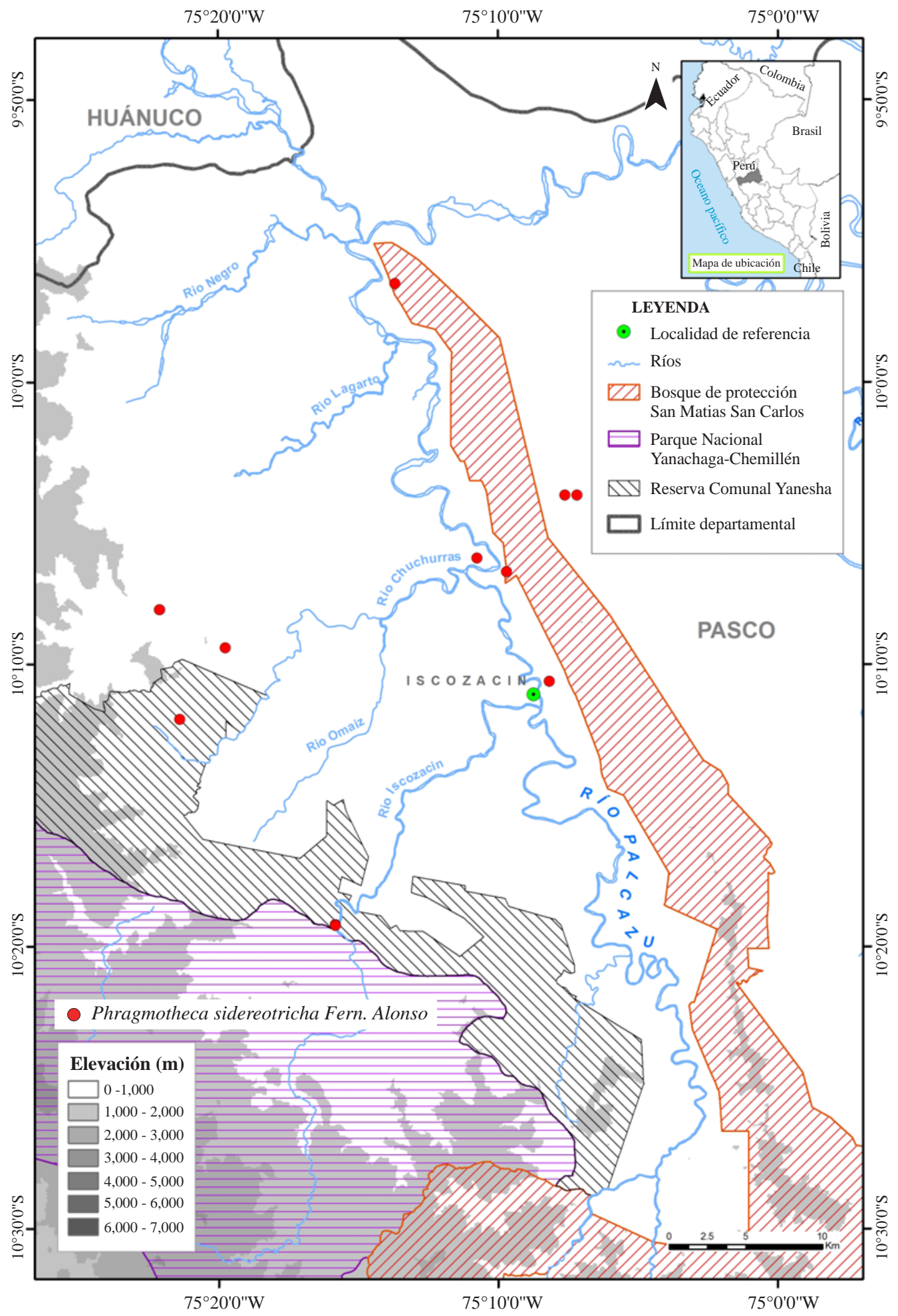

Figura 2. Mapa con la distribución conocida de Phragmotheca sidereotricha, especie endémica de la cuenca del río Palcazú, en Pasco, Perú. Las localidades aparecen señaladas con puntos rojos.

estrellado-fasciculado, mesocarpo fibroso y gelatinoso; con cinco huesos o pirenos fusiformes de (3)3,5-4 x 1,5-1,7 $\mathrm{cm}$, de consistencia leñosa, de 1,5-2,5 $\mathrm{mm}$ de grosor, cara externa fibrosa aparentemente sin proyecciones o surcos, cara interna lisa, brillante, color castaño. Semilla con testa marrón rojiza en seco, lisa, de $20-22 \mathrm{~mm}$ x $10 \mathrm{~mm}$ y de 4-4,5 mm de grosor, comprimida lateralmente, cotiledones color crema, planos (no corrugados). 
Nombres vernáculos. "Zapotillo rosado" (W. Pariona $\&$ J. Quijano 55). En cuanto a la etimología, el epíteto específico hace referencia al indumento del envés foliar constituido por tricomas estrellados con los radios dispuestos en un solo plano (Fernández-Alonso, 1996).

Distribución y hábitat. P. sidereotricha es una especie endémica de la selva central de Perú, restringida a la cuenca del río Palcazú, dentro del Bosque de Protección San Matías - San Carlos (BPSMSC) y en sus límites, en el Parque Nacional Yanachaga Chemillén (PNYCH) y en la Reserva Comunal Yanesha (RCY), todas áreas naturales protegidas (ANP) pertenecientes al Sistema Nacional de Áreas Naturales Protegidas por el Estado (SINANPE), ubicadas en la Provincia de Oxapampa, en el Departamento de Pasco, en un sector de unos $50 \times 25 \mathrm{~km}$ de área (Figura 2). Asimismo, $P$. sidereotricha presenta un rango altitudinal entre los 320 y los $700 \mathrm{msnm}$ en zonas de bosques primarios o con escasa perturbación antrópica que, según Vázquez, et al. (2005), corresponden al bosque húmedo tropical (bh-T), con una temperatura promedio de $25^{\circ} \mathrm{C}$, y se desarrollan en colinas disectadas abruptas, constituidas por arcillas y rocas expuestas. En estos bosques el dosel alcanza los $30 \mathrm{~m}$ y los árboles emergentes hasta los $40 \mathrm{~m}$ de alto.

Las zonas donde se han localizado poblaciones de $P$. sidereotricha presentan una diversidad florística relativamente alta, con árboles de troncos rectos y a menudo con aletones bien desarrollados (Vásquez, et al., 2005). Además, se tienen registros para esta región de otras siete malváceas de la tribu Matisieae: Matisia bicolor Ducke, M. cordata Bonpl., M. malacocalyx (A. Robyns \& S. Nilsson) W.S. Alverson, M. ochrocalyx K. Schum., Quararibea asterolepis Pittier, Q. guianensis Aubl. y Q. witti K. Schum. \& Ulbr. (Tropicos ${ }^{\circledR}$, 2016), representando casi un tercio del total de especies (27) reportadas o documentadas para esta tribu en Perú (Brako \& Zarucchi, 1993; Fernández-Alonso, 2001; Pennington, et al., 2004; Ulloa Ulloa, et al., 2004; Monteagudo \& Huamán, 2010; Fernández-Alonso, datos no publicados).

Ecología. Con base en todo el material de herbario revisado (26 pliegos), se pudo apreciar que tanto la floración como la fructificación de $P$. sidereotricha se presenta en dos períodos del año, la primera principalmente de mayo a julio y la segunda, de noviembre a diciembre. Asimismo, durante las evaluaciones de campo se ha podido registrar que los individuos de $P$. sidereotricha comparten el estrato intermedio y superior del bosque junto a especies como Chrysochlamys membranacea Planch. \& Triana (Clusiaceae), Dipteryx alata Vogel (Fabaceae), Eschweilera andina (Rusby) J.F. Macbr. (Lecythidaceae), Himatanthus sucuuba (Spruce ex Müll. Arg.) Woodson (Apocynaceae), Iryanthera juruensis Warb. (Myristicaceae), Miconia aff. calvescens DC. (Melastomataceae), Nealchornea yapurensis Huber (Euphorbiaceae), Otoba parvifolia (Markgr.) A.H. Gentry (Myristicaceae), Pleuranthodendron lindenii (Turcz.) Sleumer (Salicaceae), Socratea exorrhiza (Mart.) H. Wendl. (Arecaceae) y Virola sebifera Aubl. (Myristicaceae).
Como polinizadores de los géneros Matisia y Quararibea se han registrado principalmente murciélagos y, en menor medida, abejas, lepidópteros y picaflores (Alverson, 1984; Ayala, 1985, 1986; Alverson, 1989a; Valois \& Ramos, 2007; Fleming, et al., 2009; Vale, et al., 2013). Teniendo en cuenta que dentro de Matisieae la mayoría de sus especies presenta corolas blancas, amarillentas o blanco-verdosas (Alverson, 1989b; Fernández-Alonso, 1996), esta mayor afinidad del grupo hacia la quiropterofilia resulta comprensible (Faegri \& van der Pijil, 1979). Sin embargo, dentro del género Phragmotheca existen tres especies (Phragmotheca rubriflora Fern. Alonso, P. mambitana Fern. Alonso y $P$. sidereotricha) que poseen corolas de color rojo y cálices tubulares, caracteres sugerentes de polinización por colibríes, considerando lo referido en otros casos y grupos taxonómicos (Faegri \& van der Pijil, 1979; Rodríguez \& Santamaría, 2004).

En cuanto a la dispersión de semillas, en la tribu Matisieae se ha señalado repetidamente la zoocoria, encontrándose registros específicos para especies de Matisia de frutos medianos o grandes como $M$. bicolor Ducke, $M$. cordata Bonpl. y M. ochrocalyx, en las cuales los frutos son consumidos por mamíferos mayores como venados (Mazama americana Erxleben), tapires (Tapirus terrestres L.) y primates (Ateles sp.; Cebus sp.) (Reynel, et al., 2003; Palma \& Stevenson, 2013; Reynel, et al., 2016). Este sería el caso de esta especie del género Phragmotheca. Además, conocemos el caso de algunos pirenos de Matisia sp., recogidos en una clásica cueva de guácharos en Colombia, junto con otras semillas habituales en la dieta de aves de la especie Steatornis caripensis Humboldt, (Arecaceae, Lauraceae, Myrtaceae, Burseraceae, etc.). Se trataría de una dieta inusual, no formalmente documentada hasta la fecha en estas aves (Rojas, 2012; Fernández-Alonso, datos no publicados).

En el caso de especies de frutos de menor tamaño como Quararibea asterolepis, Quararibea funebris (La Llave) Vischer y Quararibea witti, se ha señalado la frugivoría por murciélagos como Artibeus jamaicensis Leach y Carollia perspicillata L. (Bonaccorso, 1979; Estrada, et al., 1984; Foster, et al., 1986; Flores, et al., 1999).

Estado de conservación. En la actualidad una gran parte de la superficie boscosa de la zona norte de la cuenca del río Palcazú ha sido transformada principalmente en áreas de pastoreo, lo que ha ocasionado la fragmentación de varios ecosistemas y la disminución de los bosques conservados, lo cual ha ocasionado que especies típicas de bosques primarios sean cada vez más difíciles de localizar. Probablemente por estas razonas casi la totalidad de las recolecciones de $P$. sidereotricha provienen de áreas naturales protegidas (BPSMSC, PNYCH y RCY) o de áreas contiguas, cuyos límites de distribución no rebasan los 50 km linealmente. Teniendo en cuenta estas características y los criterios de la UICN (2012), se optó por asignarle a esta especie la categoría de vulnerable [VU B2b (ii)]. 
Material representativo examinado. PERÚ. Dpto. Pasco, Prov. Oxapampa, Distrito Palcazú. Ataz. Camino al convento, $10^{\circ} 09^{\prime} 25^{\prime}$ 'S $75^{\circ} 19^{\prime} 45^{\prime \prime} \mathrm{O}, 652 \mathrm{msnm}, 22$ de mayo del 2009, estéril, L. Valenzuela, et al. 12792 (USM); Bosque de Protección San Matías - San Carlos, 106'43.52"S 75'9'41,68'O, $450 \mathrm{msnm}, 22$ de noviembre del 2014, fl., fr., R. Fernández-Hilario \& Policarpio 731 (HOXA, HSP, MA, MOL, USM); Bosque de Protección San Matías-San

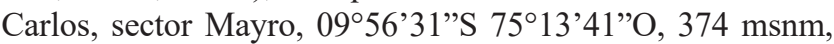
23 de mayo del 2008, fl., R. Vásquez, et al. 34249 (USM); Comunidad Nativa Alto Lagarto, $10^{\circ} 11^{\prime} 57^{\prime \prime} \mathrm{S} 75^{\circ} 21^{\prime} 23^{\prime} \mathrm{O}$, $700 \mathrm{msnm}, 03$ de diciembre del 2007, fr., R. Rojas, et al. 4890 (USM); Comunidad Nativa Alto Lagarto - Reserva Comunal Yanesha, $10^{\circ} 08^{\prime} 04^{\prime \prime} \mathrm{S} 75^{\circ} 22^{\prime} 06^{\prime}$ 'O, $500 \mathrm{msnm}, 19$ de diciembre del 2008, fl., R. Rojas \& G. Ortiz 6394 (USM); Comunidad Nativa Alto Lagarto - Reserva Comunal Yanesha, $10^{\circ} 08^{\prime} 04^{\prime \prime S} 75^{\circ} 22^{\prime} 06^{\prime}$ O, 500 msnm, 02 de julio del 2009, fr., R. Rojas \& G. Ortiz 6783 (USM); Iscozacín, sector Cuacuazú, $10^{\circ} 10^{\prime} 36.63$ "S $75^{\circ} 8^{\prime} 9,83^{\prime \prime} \mathrm{O}, 320 \mathrm{msnm}, 16$ de febrero del 2015, estéril, R. Fernández-Hilario s/n (HSP); Límite del Parque Nacional Yanachaga - Chemillén, cerca de la estación biológica Paujil, 10¹9'15.4”S 75¹5'48,9”O, 390 msnm, 22 de junio del 2005, fr., A. Monteagudo, et al. 9171 (HOXA, MA, MO); Parque Nacional Yanachaga - Chemillén; trocha de la estación biológica Paujil, $10^{\circ} 19^{\prime} 14^{\prime \prime S} 75^{\circ} 15^{\prime} 49^{\prime \prime O}$, $420 \mathrm{msnm}, 24$ de mayo del 2003, fr., A. Monteagudo, et al. 5472 (AMAZ, HOXA, MA, MO); Parque Nacional Yanachaga - Chemillén, cruzando el río Iscozacín, parcela Paujil $1 \mathrm{Ha}, 10^{\circ} 20^{\prime} \mathrm{S} 75^{\circ} 15^{\prime} \mathrm{O}, 410 \mathrm{~m}$, fl., 14 de julio del 2007, Monteagudo, et al. 14255 (COL, MO); zona del río San José, drenaje del río Chuchurras, 400-500 m, 1009'S 75²0'O, pendientes con suelos calizos, 14 de mayo de 1983, fr., $D$. $N$. Smith 4029A (MO). Distrito Puerto Bermúdez. CC. NN. 24 de

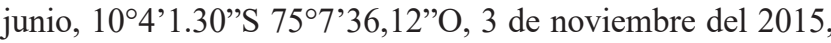
estéril, J. Marcelo-Peña 10205 (MOL); CC. NN. 24 de junio, $10^{\circ} 4^{\prime} 1.15^{\prime \prime} \mathrm{S} 75^{\circ} 7^{\prime} 10,59^{\prime \prime} \mathrm{O}, 3$ de noviembre del 2015, estéril, J. Marcelo-Peña 10210 (MOL).

\section{Contribución de los autores}

Introducción, género y clave de géneros, por: J.L. Fernández, R. Fernández y C. Reynel

Descripción de la especie nueva y colecciones revisadas, por: J.L. Fernández y R. Fernández

Distribución, hábitat, ecología, conservación e ilustraciones, por: R. Fernández, C. Reynel y J.L. Fernández.

\section{Agradecimientos}

A los curadores de los herbarios COL, HOXA y USM por las facilidades brindadas durante la revisión de material, y al herbario MO por el envío de préstamos y duplicados de Malvaceae para la determinación. A Andrea Bravo por la elaboración del mapa de distribución. A los revisores de este manuscrito por las oportunas observaciones realizadas.

\section{Conflicto de intereses}

Los autores declaran no tener conflictos de intereses.

\section{Referencias}

Alverson, W. (1984). Quararibea pumila (Bombacaceae), a new endemic from Costa Rica. Brittonia. 36 (3): 252-256. https:// doi.org/10.2307/2806520

Alverson, W. (1989a). Quararibea (Bombacaceae): Five new species from moist and wet forest of Costa Rica and Panamá. Brittonia. 41 (1): 61-74. https://doi.org/10.2307/2807592

Alverson, W. (1989b). Matisia and Quararibea (Bombacaceae) should be retained as separate genera. Taxon. 38 (3): $377-$ 388. https://doi.org/10.2307/1222268

Alverson, W. (1991). A synopsis of Phragmotheca (Bombacaceae), with two new species and a new subspecies. Brittonia. 43 (2): 73-87. https://doi.org/10.2307/2807297

Alverson, W., Whitlock, A., Nyffeler, R., Bayer, C., Baum, D. (1999). Phylogeny of the core Malvales: Evidence from ndhF sequence data. American Journal of Botany. 86 (10): 1474-1486.

Angiosperm Phylogeny Group - APG IV. (2016). An update of the Angiosperm Phylogeny Group classification for the orders and families of flowering plants: APG IV. Botanical Journal of the Linnean Society. 181 (1): 1-20. https://doi. org/10.1111/boj. 12385

Ayala, A.V. (1985). Observaciones entre las interacciones del colibrí Thalurania furcate colombica Bourcier, y las flores que les sirven de fuente de alimento en un bosque subandino. Tesis de Biología, Universidad Nacional de Colombia.

Ayala, A.V. (1986). Aspectos de la relación entre Thalurania furcata colombica (Aves, Trochilidae) y las flores en que liba, en un bosque subandino. Caldasia. 14 (68-70): 549-562.

Baum, D., Smith, S., Yen, A., Alverson, W., Nyffeler, R., Whitlock, B., Oldham, R. (2004). Phylogenetic relationships of Malvatheca (Bombacoideae and Malvoideae; Malvaceae sensu lato) as inferred from plastid DNA sequences. American Journal of Botany. 91 (11): 1863-1871. https:// doi.org/10.3732/ajb.91.11.1863

Bayer, C., Fay, M., Brujin, A., Savolainen, V., Morton, C., Kubitzki, K., Alverson, W., Chase, M. (1999). Support for an expanded family concept of Malvaceae within a recircumscribed order Malvales: A combined analysis of plastid atpB and rbcL DNA sequences. Botanical Journal of the Linnean Society. 129 (4): 267-303. https://doi.org/10. $1111 /$ j.1095-8339.1999.tb00505.x

Bayer, C., Kubitzki, K. (2003). Malvaceae. En: K. Kubitzki, C. Bayer. (Eds.). The families and genera of vascular plants. Flowering plants, dicotyledons V: Malvales, Capparales and non-betalain Caryophyllales. Berlin, Alemania: Springer. p. 225-311.

Brako, L., Zarucchi, J. (1993). Catalogue of the Flowering Plants and Gymnosperms of Perú. Monogr. Syst. Bot. Missouri Bot. Garden. 45: 1-1286.

Bonaccorso, F. (1979). Foraging and reproductive ecology in a Panamanian bat community. Bulletin of the Florida State Museum, Biological Sciences. 24 (4): 359-408.

Carvalho-Sobrinho, J., Alverson, W., Alcantara, S., Queiroz, L., Mota, A., Baum, D. (2016). Revisiting the phylogeny of Bombacoideae (Malvaveae): Novel relationships, morphologically cohesive clades, and a new tribal classification based on multilocus phylogenetic analyses. Molecular Phylogenetics and Evolution. 101: 56-74. https://doi.org/10. 1016/j.ympev.2016.05.006 
Duarte, M., Esteves, G., Salatino, M., Walsh, K., Baum, D. (2011). Phylogenetic analyses of Eriotheca and related genera (Bombacoideae, Malvaceae). Systematic Botany. 36 (3): 690-701. https://doi.org/10.1600/036364411X583655

Estrada, A., Coates, R., Vásquez, C., Orozco, A. (1984). Comparison of frugivory by howling monkeys (Alouatta palliata) and bats (Arbiteus jamaicensis) in the tropical rain forests of Los Tuxtlas, México. American Journal of Primatology. 7: 3-13. https://doi.org/10.1002/ajp.1350070103

Faegri, K., van der Pijil, L. (1979). The principles of pollination ecology. Pergamon Press, Oxford. p. 244.

Fernández-Alonso, J.L. (1996). Contribuciones al conocimiento del género Phragmotheca Cuatr. (Bombacaceae-Quararibeae). Rev. Acad. Colomb. Cienc. 18 (3): 253-284.

Fernández-Alonso, J.L. (1999). Nueva especies de zapote de monte (Phragmotheca, Bombacaceae) en bosques premontanos del centro de Colombia. Caldasia. 21 (2): 125-131.

Fernández-Alonso, J.L. (2001). Bombacaceae neotropicae novae vel minus cognitae I. Novedades taxonómicas y corológicas en Matisia, Quararibea y Spirotheca. Rev. Acad. Colomb. Cienc. 25 (95): 183-206.

Fernández-Alonso, J.L. (2011). Bombacaceae neotropicae novae vel minus cognitae IX. Una nueva especie de Matisia Bonpl. del Chocó biogeográfico. Caldasia. 33 (2): 393-406.

Fleming, T., Geiselman, C., Kress, J. (2009). The evolution of bat pollination: A phylogenetic perspective. Annals of Botany. 104: 1017-1043. https://doi.org/10.1093/aob/mcp197

Flores, J., Ortega, J., Ibarra, G. (1999). El hábito alimentario del murciélago zapotero (Artibeus jamaicensis) en Yucatán. Revista Mexicana de Mastozoología. 4: 22-39.

Foster, R., Arce, J., Wachter, T. (1986). Dispersal and the sequential plant communities in Amazonian Perú floodplain. En: A. Estrada, T. Fleming (Eds.). Frugivores and seed dispersal. Dordrecht, Holanda: Dr W. Junk Publishers. p. 357-370.

Monteagudo, A., Huamán, M. (2010). Catálogo de los árboles y afines de la Selva Central del Perú. Arnaldoa. 17 (2): 203242.

Nyffeler, R., Bayer, C., Alverson, W., Yen, A., Whitock, B., Chase, M., Baum, D. (2005). Phylogenetic analysis of the Malvadendrina clade (Malvaceae s.1.) based on plastid DNA sequences. Organisms, Diversity \& Evolution. 5: 109-123. https://doi.org/10.1016/j.ode.2004.08.001

Palma, A., Stevenson, P. (2013). Dispersión de semillas por monos araña en la Estación Biológica Cocha Cashu, Parque Nacional Manu, Perú. En: J. Groenendijk, A. Tovar, W. Wust. (Eds). Reporte Manu 2013: Pasión por la Investigación en la Amazonía Peruana (178-195). San Diego Zoo Global Peru \& SERNANP.
Pennington, T. D., Reynel, C., Daza, A. (2004). Illustrated guide to the tree of Perú. David Hunt, The Manse, Chapel Lane, Milborne Port Sherborne, England. p. 848.

Reynel, C. Pennington, R., Pennington, T., Flores, C., Daza, A. (2003). Árboles útiles de la Amazonía peruana y sus usos: un manual con apuntes de identificación, ecología y propagación de las especies. Herbario de la Facultad de Ciencias Forestales de la Universidad Nacional Agraria La Molina, Royal Botanic Gardens Kew, Royal Botanic Gardens Edinburgh e ICRAF. p. 537.

Reynel, C., Pennington, T., Pennington, R. (2016). Árboles del Perú. Herbario de la Facultad de Ciencias Forestales de la Universidad Nacional Agraria La Molina, The Natural Environment Research Council, Royal Botanic Gardens Edinburgh, Darwin Initiative, Centro de Estudios en Dendrología - Fundación para el Desarrollo Agrario \& Asociación Peruana para la Promoción del Desarrollo Sostenible. p. 1047.

Rodríguez, M., Santamaría, L. (2004). Why are so many bird flowers red? PLOS Biology. 2 (10): 1515-1519. https://doi. org/10.1371/journal.pbio.0020350

Rojas Lizarazo, G.L. (2012). Dieta del guácharo Steatornis caripensis Humboldt, 1817 (Aves: Steatornithidae) en el Parque Nacional Natural Chingaza, Cundinamarca Colombia. Tesis de Postgrado en Biología. Universidad Nacional de Colombia. p. 81.

Tropicos. (2016). Base de datos de especímenes (en línea). Missouri, USA. Disponible en http://www.tropicos.org/ SpecimenSearch.aspx. Fecha de acceso: julio de 2016.

UICN. (2012). Categorías y criterios de la Lista Roja de la UICN: Versión 3.1. Segunda edición. Gland, Suiza y Cambridge, Reino Unido: UICN. p. 34.

Ulloa Ulloa, C., Zarucchi, J., León, B. (2004). Diez años de adiciones a la flora del Perú: 1993-2003. Arnaldoa. Número especial: 1-242.

Vale, V., Schiavini, I., Lopes, S., Oliveira, A., Dias, O., Gusson, A. (2013). Functional groups in a semideciduous seasonal forest in Southeastern Brazil. Biotemas. 26 (2): 45-58. https://doi.org:10.5007/2175-7925.2013v26n2p45

Valois, H., Ramos, Y. (2007). Bombacaceae: Composition and ecology in permanent plot of research on biodiversity (PPRB), Chocó, Colombia. Revista Institucional Universidad Tecnológica del Chocó: Investigación, Biodiversidad y Desarrollo. 26 (2): 4-8.

Vázquez, R., Rojas, R., Monteagudo, A., Meza, K., van der Werff, H., Ortiz, R., Catchpole, D. (2005). Flora vascular de la selva central del Perú: una aproximación a la composición florísticas de tres Áreas Naturales Protegidas. Arnaldoa. 12 (1-2): 112-125. 